\title{
Detection-Localization Tradeoff in MIMO Radars
}

\author{
Mohammad NAZARI MAJD, Mojtaba RADMARD, Mohammad M. CHITGARHA, \\ Mohammad H. BASTANI, Mohammad M. NAYEBI \\ Dept. of Electrical Engineering, Sharif University of Technology, Tehran, Iran \\ \{nazarimajd, radmard, chitgarha\}@ee.sharif.ir, \{bastanih, nayebi\}@ sharif.ir \\ Submitted September 6, 2016 / Accepted January 2, 2017
}

\begin{abstract}
Two gains play key roles in recently developed MIMO wireless communication systems: "spatial diversity" gain and "spatial multiplexing" gain. The diversity gain refers to the capability to decrease the error rate of the MIMO channel, while the multiplexing gain implicitly refers to the amount of increase in the capacity of the MIMO channel. It has been shown that there is a fundamental tradeoff between these two types of gains, meaning interplay between increasing reliability (via an increase in the diversity gain) and increasing data rate (via an increase in the multiplexing gain). On the other hand, recently, MIMO radars have attracted much attention for their superior ability to enhance the system's performance. As a MIMO system, it is expected that the mentioned diversity-multiplexing tradeoff exists in a MIMO radar, too. In this paper, this tradeoff is studied and verified in MIMO radars with widely separated antennas. In more details, it will be shown that increasing dependency between transmit-receive links results in higher diversity gain and lower multiplexing gain, and vice versa. Then, the optimal tradeoff is introduced, i.e., the conditions that the MIMO radar system should have, so that the diversity-multiplexing tradeoff is at its optimum point, are driven.
\end{abstract}

\section{Keywords}

MIMO radar, widely separated antennas, detection, localization, tradeoff

\section{Introduction}

After the spark in communication leading to the popular and widely noticed Multiple-Input Multiple-Output communications, the idea of Multiple-Input Multiple-Output (MIMO) radar was also developed. Generally, MIMO radar can be categorized as systems with widely separated antennas or systems that use colocated antennas. In the colocated structure, the antennas are placed closely together [1]. On the other hand, in the case of widely separated antennas (WSMIMO), multiple transmitters and multiple receivers that are widely separated are used. The studies show that widely separated antennas scheme can provide enhanced detection performance (diversity gain) [2-4] and high resolution target localization (spatial multiplexing gain) [5], [6].
In this paper, we focus on the MIMO radars with widely separated antennas. As shown in [7], the concepts of spatial diversity and multiplexing gain emerge in this context in a dual manner to the MIMO communication. The main point is that, by looking at a target from different angles, the probability of missed detection decreases, a concept known as spatial diversity in MIMO communication. In [8], it is shown that such diversity gain is available not only in the signal processing part but also in the data processing part. Indeed, the target's Radar Cross Section in MIMO radars plays the role of the communication channel in MIMO communication, that can fade resulting in missed detection.

In wireless communication systems, multiple antennas can be used to achieve diversity gain or multiplexing gain. In [9], the authors proposed the point of view that both types of gains can be simultaneously obtained for a given multiple antenna channel, but there is a fundamental tradeoff between how much of each any coding scheme can get. Then, they introduce optimal diversity-multiplexing tradeoff (DMT) as a benchmark to compare the various space-time schemes. Since then, numerous papers were published on this topic in communication (e.g. see [10-13]).

According to the aforementioned dualities between a MIMO radar system and a MIMO wireless communication system, it is expected that such tradeoff exists in a MIMO radar, too. In [14], it is stated that such tradeoff should exist and analyzing this tradeoff is considered as a long-term goal. Moreover, in [15], irrespective of multiplexing gain, the interplay between transmit diversity and energy integration in a MIMO radar is studied for a Generalized Likelihood Ratio Test based detector, and it is shown that there is an inherent tradeoff between the number of transmit diversity paths and amount of energy integration along each path.

Before investigating such tradeoff, first, it is necessary to introduce the concepts of diversity and multiplexing gain in a MIMO radar. It has been shown in the MIMO radar literature that the diversity gain appears in the probability of missed detection [7], similar to the MIMO communication where it appears in the error probability. Thus the diversity gain of a MIMO radar system can be defined as [16]

$$
\mathrm{DG}=-\lim _{\mathrm{SNR} \rightarrow \infty} \frac{\log P_{\text {miss }}}{\log \mathrm{SNR}}
$$


where $P_{\text {miss }}$ is the probability of missed detection and DG denotes diversity gain.

On the other hand, in [7], it is mentioned that the number of targets that can be handled simultaneously by a MIMO radar parallels the concept of spatial multiplexing in communications. Equivalently, this gain can be expressed in terms of the resolution capability of the MIMO radar. Indeed, exploiting the multiplexing gain, MIMO radars can locate targets with high resolution and can resolve between closely spaced targets [7].

According to the mentioned subjects, in the following, our goal is to explore the tradeoff between the diversity gain that appears in the probability of missed detection, and the multiplexing gain that appears in the resolution capability of the MIMO radar with widely separated antennas.

The rest of the paper is organized as follows. Section 2 explains the signal model used throughout the paper. The diversity and multiplexing gains of the WS-MIMO radar are studied in Sec. 3 and 4, respectively. The tradeoff existing between these two gains is introduced and verified in Sec. 5 . Section 6 is dedicated to the simulations and numerical experiments. Finally, Sec. 7 concludes the paper.

\section{Signal Model}

Consider a MIMO radar system with $N_{\mathrm{I}}$ transmit antennas and $N_{\mathrm{R}}$ receive antennas. Denoting the received signal at the $r$-th receiver by $z_{r}(t)$, the hypothesis test can be written as

$$
\left\{\begin{aligned}
\mathcal{H}_{0}: z_{r}(t) & =n_{r}(t), \\
\mathcal{H}_{1}: z_{r}(t) & =n_{r}(t)+\sum_{i=1}^{N_{\mathrm{I}}} \alpha_{r i} s_{i}\left(t-\tau_{r i}\right) \mathrm{e}^{\mathrm{j} 2 \pi f_{r i}^{d} t}, \\
r & =1,2, \ldots, N_{\mathrm{R}},
\end{aligned}\right.
$$

where $n_{r}(t)$ and $s_{i}(t)$ are the $r$-th receiver noise and $i$-th transmit signal, respectively. In addition, $\alpha_{r i}, \tau_{r i}$ and $f_{r i}^{d}$ are complex envelope, delay and Doppler experienced by the signal on the $i$-th transmitter-target- $r$-th receiver path (or equivalently $i-r$ link), respectively. Assuming a Swerling I model for the target, the amplitude of $\alpha_{r i}$ has a Rayleigh distribution and its phase of $\alpha_{r i}$ has a uniform distribution on $[0,2 \pi)$. Thus, $\alpha_{r i} \sim \mathcal{C N}\left(0, \sigma_{r i}^{2} I\right)$, with

$$
\sigma_{r i}^{2}=\frac{P_{i} G_{i}^{\mathrm{I}} G_{r}^{\mathrm{R}} \lambda_{i}^{2} \sigma_{0}^{2}}{(4 \pi)^{3}\left(\ell_{i}^{\mathrm{I}}\right)^{2}\left(\ell_{r}^{\mathrm{R}}\right)^{2}}
$$

where $P_{i}, G_{i}^{\mathrm{I}}, \lambda_{i}$ and $\ell_{i}^{\mathrm{I}}$ are the $i$-th transmit power, antenna gain, signal's wavelength and $i$-th transmitter to the target distance, respectively. Also, $G_{r}^{\mathrm{R}}$ and $\ell_{r}^{\mathrm{R}}$ are the $r$-th receive antenna gain and $r$-th receiver to the target distance, respectively. Moreover, $\sigma_{0}^{2}$ is the variance of target's Radar Cross Section (RCS). By defining

$$
y_{r i}(t) \triangleq s_{i}\left(t-\tau_{r i}\right) \mathrm{e}^{\mathrm{j} 2 \pi f_{r i}^{d} t},
$$

and then sampling the received signal we have

$$
\left\{\begin{array}{l}
\mathcal{H}_{0}: \mathbf{z}_{r}=\mathbf{n}_{r} \\
\mathcal{H}_{1}: \mathbf{z}_{r}=\mathbf{n}_{r}+\sum_{i=1}^{N_{\mathrm{I}}} \alpha_{r i} \mathbf{y}_{r i}, \quad r=1,2, \ldots, N_{\mathrm{R}}
\end{array}\right.
$$

In the following, noise is assumed to be white with covariance matrix $\sigma_{n}^{2} \mathbf{I}$. In addition, in our MIMO radar system with widely separated antennas, in order to obtain the amount of correlation between different RCS values viewed from different aspect angles, we assume the model proposed in [2]. There, for each pair of RCS values of $\alpha_{i r}, \alpha_{p q}\left(1 \leq i, p \leq N_{\mathrm{I}}, 1 \leq r, q \leq N_{\mathrm{R}}\right)$, it is deduced that under certain conditions, which implicitly state that the antennas should be widely separated enough, $\alpha_{i r}$ and $\alpha_{p q}$ are uncorrelated, otherwise they are fully correlated. Finally, define

$$
\boldsymbol{\alpha} \triangleq\left[\begin{array}{lllllll}
\alpha_{11} & \alpha_{12} & \ldots & \alpha_{1 N_{\mathrm{R}}} & \alpha_{21} & \ldots & \alpha_{N_{\mathrm{I}} N_{\mathrm{R}}}
\end{array}\right]^{T}
$$

and

$$
\mathbf{C}_{N_{\mathrm{I}} N_{\mathrm{R}} \times N_{\mathrm{I}} N_{\mathrm{R}}}=\mathbb{E}\left\{\boldsymbol{\alpha} \boldsymbol{\alpha}^{H}\right\} .
$$

\section{Diversity Gain and MIMO Detector}

The Neyman-Pearson detector compares the likelihood ratio with a threshold value which is determined by the false alarm probability [17]. Thus, this ratio should be computed first. By observing the vector $\mathbf{z}$ which contains all receivers vectors, the $\mathcal{H}_{0}$ and $\mathcal{H}_{1}$ hypotheses can be obtained as follows

$$
T=\log \frac{f_{\mathbf{z}}\left(\mathbf{z} \mid \mathcal{H}_{1}\right)}{f_{\mathbf{z}}\left(\mathbf{z} \mid \mathcal{H}_{0}\right)} \gtrless \eta_{0} .
$$

In order to achieve the detector structure, let $\mathbf{x}$ be the vector that its $(r-1) N_{\mathrm{I}}+i$ element is

$$
\{\mathbf{x}\}_{(r-1) N_{\mathrm{I}}+i}=\int z_{r}(t) s_{i}\left(t-\tau_{i r}\right) \mathrm{d} t=<\mathbf{z}_{r}, \mathbf{s}_{r i}>
$$

where $z_{r}(t)$ is the $r$-th receiver's signal and $\tau_{i r}$ denotes the delay that the $i$-th transmit signal, $s_{i}(t)$, experiences while being reflected by the target and received at the $r$-th receiver. In addition, the vectors $\mathbf{s}_{r i}$ and $\mathbf{z}_{r}$ contain the samples of $s_{i}\left(t-\tau_{r i}\right)$ and $z_{r}(t)$. Indeed, the elements of $\mathbf{x}$ are the outputs of the filters matched to the transmit signals at all receivers and have a size of $\left(N_{\mathrm{I}} N_{\mathrm{R}} \times 1\right)$. As shown in [2], when noise and signal powers are known, $\mathbf{x}$ is sufficient statistics for detection. Moreover, it is assumed that the transmit signals are orthonormal to each other ${ }^{1}$, i.e.,

$$
\int s_{i}(t) s_{j}(t) \mathrm{d} t=E . \delta_{i j}, \quad 1 \leq i, j \leq N_{\mathrm{I}}
$$

where $E$ is the signal's energy and $\delta$ is the Dirichlet function. The Probability Density Function (PDF) of the received vector in the $\mathcal{H}_{1}$ case can be written as

\footnotetext{
${ }^{1}$ This assumption is common in the WS-MIMO radar's literature, e.g. see [7].
} 


$$
\begin{aligned}
& f_{\mathbf{z}}\left(\mathbf{z} \mid \mathcal{H}_{1}\right)= \\
& \int f_{\mathbf{z}}\left(\mathbf{z} \mid \mathcal{H}_{1}, \boldsymbol{\alpha}\right) f_{\boldsymbol{\alpha}}(\boldsymbol{\alpha}) \mathrm{d} \boldsymbol{\alpha}=\frac{1}{\left(\sqrt{2 \pi \sigma_{n}^{2}}\right)^{N_{\mathrm{I}} N_{\mathrm{R}}}} \times \\
& \int \exp \left\{-\frac{1}{2 \sigma_{n}^{2}} \sum_{r=1}^{N_{\mathrm{R}}}\left(\left\|\mathbf{z}_{r}\right\|^{2}-2 \sum_{i=1}^{N_{\mathrm{I}}} \operatorname{Re}\left\{\alpha_{r i} \mathbf{z}_{r}^{H} \mathbf{y}_{r i}\right\}+\right.\right. \\
& \left.\left.\sum_{i=1}^{N_{\mathrm{I}}} E^{2}\left|\alpha_{r i}\right|^{2}\right)\right\} \times f_{\boldsymbol{\alpha}}(\boldsymbol{\alpha}) \mathrm{d} \boldsymbol{\alpha} .
\end{aligned}
$$

Now, the RCSs of $N_{\mathrm{I}} N_{\mathrm{R}}$ different paths (or equivalently transmit-receive links) can be divided to $\zeta$ subsets, such that the RCSs in each subset are fully correlated with each other, but completely independent of the RCSs in other subsets. Let $T_{m}$ be the number of correlated links included in the $m$ 'th subset. So,

$$
\begin{aligned}
& f_{\mathbf{z}}\left(\mathbf{z} \mid \mathcal{H}_{1}\right)=\frac{1}{\left(\sqrt{2 \pi \sigma_{n}^{2}}\right)^{N_{\mathrm{I}} N_{\mathrm{R}}}} \mathrm{e}^{-\frac{1}{2 \sigma_{n}^{2}} \sum_{r=1}^{N_{\mathrm{R}}}\left\|\mathbf{z}_{r}\right\|^{2}} \times \\
& \prod_{m=1}^{\zeta} \int \mathrm{e}^{-\frac{1}{2 \sigma_{n}^{2}}\left(-2 \sum_{k=1}^{T_{m}} \operatorname{Re}\left\{\alpha_{m} x_{m k}\right\}-T_{m} E^{2}\left|\alpha_{m}\right|^{2}\right)} \mathrm{e}^{-\frac{\left|\alpha_{m}\right|^{2}}{\sigma_{\alpha_{m}}^{2}}} \mathrm{~d} \alpha_{m} \\
& =k \mathrm{e}^{-\frac{1}{2 \sigma_{n}^{2}} \sum_{r=1}^{N_{\mathrm{R}}}\left\|\mathbf{z}_{r}\right\|^{2}} \prod_{m=1}^{\zeta} \exp \left\{\frac{\left|\sum_{k=1}^{T_{m}} x_{m k}\right|^{2}}{2 \sigma_{n}^{2} / \sigma_{\alpha_{m}}^{2}+T_{m} E^{2}}\right\}
\end{aligned}
$$

For $\mathcal{H}_{0}$ hypothesis, the received vector contains noise only and

$$
f_{\mathbf{z}}\left(\mathbf{z} \mid \mathcal{H}_{0}\right)=\frac{1}{\left(\sqrt{2 \pi \sigma_{n}^{2}}\right)^{N_{\mathrm{I}} N_{\mathrm{R}}}} \mathrm{e}^{-\frac{1}{2 \sigma_{n}^{2}} \sum_{r=1}^{N_{\mathrm{R}}}\left\|\mathbf{z}_{r}\right\|^{2}} .
$$

Subsequently, the loglikelihood ratio can be simplified as

$$
\begin{aligned}
T & =\log f_{\mathbf{z}}\left(\mathbf{z} \mid \mathcal{H}_{1}\right)-\log f_{\mathbf{z}}\left(\mathbf{z} \mid \mathcal{H}_{0}\right) \\
& =\sum_{m=1}^{\zeta} \frac{\left|\sum_{k=1}^{T_{m}} x_{m k}\right|^{2}}{2 \sigma_{n}^{2} / \sigma_{\alpha_{m}}^{2}+T_{m} E^{2}} \gtrless \eta_{1} .
\end{aligned}
$$

Defining $q_{m}$ as

$$
q_{m} \triangleq\left|\sum_{k=1}^{T_{m}} x_{m k}\right|^{2},
$$

$T$ will be a linear combination of $q_{m}$ s which have Chi square distribution. It is shown that the probability that a linear summation of Chi square random variables weighted with $c_{i}$ coefficients (i.e. $\left.W=\sum_{i=1}^{p} c_{i} \chi^{2}\left(n_{i}\right)\right)$ has the following Cumulative Distribution Function (CDF) [18]

$$
F_{W}(w)=\left(\prod_{i=2}^{p} b_{i}\right) \sum_{j=0}^{\infty} a_{j} \int_{0}^{w} g_{j}(y) \mathrm{d} y
$$

where

$$
\begin{aligned}
g_{j}(y) & =y^{s+j-1} \frac{\mathrm{e}^{-\frac{y}{2 c_{1}}}}{\left(2 c_{1}\right)^{s+j} \Gamma(s+j)}, \\
a_{j} & =A_{j}^{(p)}, \\
A_{j}^{(i)} & =\sum_{k=0}^{j} A_{k}^{(i-1)} u\left(c_{i}, j-k\right)
\end{aligned}
$$

and

$$
b_{i}=\left(\frac{c_{1}}{c_{i}}\right)^{m_{i}}
$$

$$
\begin{aligned}
u\left(c_{i}, r\right) & =\left(m_{i}\right)_{r} \frac{\left(1-\frac{c_{1}}{c_{i}}\right)^{r}}{r !}, \\
(m)_{r} & =m(m+1), \ldots,(m+r-1), \\
s & =\sum_{i=1}^{p} m_{i}, \\
m_{i} & =\frac{n_{i}}{2} .
\end{aligned}
$$

In our problem $q_{m}=\left|\sum_{k=1}^{T_{m}} x_{m k}\right|^{2}$ has a Chi square distribution with two degrees of freedom $\left(n_{i}=2\right)$, since all $T_{m}$ signals of the $m$ 'th subset are fully correlated. So,

$$
s=\sum_{i=1}^{p} m_{i}=\sum_{i=1}^{\zeta} 1=\zeta .
$$

After some calculation, diversity gain (or the slope of $\log P_{\text {miss }}$ at high SNR values) is equal to $\zeta$ (i.e., the number of independent subsets constituted from the $N_{\mathrm{I}} N_{\mathrm{R}}$ links). So,

$$
\mathrm{DG}=\zeta
$$

\section{Localization Accuracy and Multi- plexing Gain}

In this section, the multiplexing order is obtained. As mentioned before, the multiplexing gain is defined as the resolution capability. Moreover, the resolution is directly related to the localization accuracy [19]. Thus, we can deduce that improvement of this accuracy is proportional to the multiplexing gain. In the sequel, in order to find the localization accuracy, first, Geometric Dilution of Precision (GDOP), which is a criterion of localization accuracy, is computed. Then, multiplexing order is defined by GDOP.

The GDOP parameter, which is a representative of the localization error, is defined as [20]

$$
\begin{aligned}
\text { GDOP } & =\frac{1}{\sigma_{R}} \operatorname{tr}\left\{\mathbf{C}_{\text {BLUE }}\right\} \\
& =\frac{1}{\sigma_{R}} \operatorname{tr}\left\{\left(\mathbf{D}^{T} \Sigma^{-1} \mathbf{D}\right)^{-1}\right\}
\end{aligned}
$$

where $\sigma_{R}$ is the variance of the bistatic range and $\mathbf{D}$ is the transfer function obtained from 


$$
\boldsymbol{\tau}_{N_{\mathrm{I}} N_{\mathrm{R}} \times 1}=\mathbf{D}_{N_{\mathrm{I}} N_{\mathrm{R}} \times 2} \boldsymbol{\theta}_{2 \times 1}
$$

where $\boldsymbol{\theta}=[x, y]^{T}$ and $\boldsymbol{\tau}$ contain the target's position in the Cartesian coordinates, and the received signals' delays due to such target, respectively. Also it is assumed that estimated delay is a Gaussian random variable with covariance matrix of

$$
\begin{aligned}
& \boldsymbol{\Sigma}_{N_{\mathrm{I}} N_{\mathrm{R}} \times N_{\mathrm{I}} N_{\mathrm{R}}}=\left[\begin{array}{ccc}
\mathbb{E}\left\{\tau_{11} \tau_{11}\right\} & \ldots & \mathbb{E}\left\{\tau_{11} \tau_{N_{\mathrm{I}} N_{\mathrm{R}}}\right\} \\
\vdots & \ddots & \vdots \\
\mathbb{E}\left\{\tau_{N_{\mathrm{I}} N_{\mathrm{R}}} \tau_{11}\right\} & \ldots & \mathbb{E}\left\{\tau_{N_{\mathrm{I}} N_{\mathrm{R}}} \tau_{N_{\mathrm{I}} N_{\mathrm{R}}}\right\}
\end{array}\right] \\
& =\left[\begin{array}{ccc}
\frac{\rho_{11,11}}{\mathrm{SNR}_{11}} & \cdots & \frac{\rho_{11, N_{\mathrm{I}} N_{\mathrm{R}}}}{\sqrt{\mathrm{SNR}_{11} \mathrm{SNR}_{N_{\mathrm{I}} N_{\mathrm{R}}}}} \\
\vdots & \ddots & \vdots \\
\frac{\rho_{N_{\mathrm{I}} N_{\mathrm{R}}, 11}}{\sqrt{\mathrm{SNR}_{N_{\mathrm{I}} N_{\mathrm{R}}} \mathrm{SNR}_{11}}} & \cdots & \frac{\rho_{N_{\mathrm{I}} N_{\mathrm{R}}, N_{\mathrm{I}} N_{\mathrm{R}}}}{\mathrm{SNR}_{N_{\mathrm{I}} N_{\mathrm{R}}}}
\end{array}\right]
\end{aligned}
$$

where $\mathrm{SNR}_{i j}$ is the signal to noise ratio of the $i$-th transmitted signal that is received at the $r$-th receiver. Morever, $\rho_{i j, i^{\prime} j^{\prime}}$ represents the amount of normalized correlation between $\tau_{i j}$ and $\tau_{i^{\prime} j^{\prime}}$.

As explained before, the multiplexing gain has a close relation to the localization accuracy. Thus, herein, we define the multiplexing gain as

$$
\mathrm{MG} \triangleq \lim _{\mathrm{SNR} \rightarrow \infty} \frac{G}{\mathrm{SNR}}
$$

where $\mathrm{SN} R$ is the average of all $\mathrm{SNR}_{i j}, 1 \leq i \leq N_{\mathrm{I}}, 1 \leq j \leq$ $N_{\mathrm{R}}$, and $G=\kappa \cdot \mathrm{GDOP}^{-1}$. Here, $\kappa$ is chosen such that in the case of full diversity, the multiplexing gain equals one.

\section{Optimal Tradeoff Between Diversity and Multiplexing Gain}

In this section we investigate the tradeoff that may exist between the two MIMO gains of a WS-MIMO radar, i.e., the diversity gain and the multiplexing gain.

Consider a WS-MIMO radar system, in which $N_{\mathrm{I}} N_{\mathrm{R}}$ number of RCS values are seen from different target's aspect angles. In Sec. 3, it was shown that the diversity order of such system equals to the number of independent subsets $(\zeta)$ constituted from the $N_{\mathrm{I}} N_{\mathrm{R}}$ links. Moreover, the multiplexing order was defined in Sec. 4, using the localization precision of the MIMO system. Accordingly, the two following cases can be considered.

Consider a WS-MIMO radar system, in which $N_{\mathrm{I}} N_{\mathrm{R}}$ number of RCS values are seen from different target's aspect angles. In Sec. 3, it was shown that the diversity order of such system equals to the number of independent subsets $(\zeta)$ constituted from the $N_{\mathrm{I}} N_{\mathrm{R}}$ links. Moreover, the multiplexing order was defined in Sec. 4, using the localization precision of the MIMO system. It can be seen in (21) that if the number of independent subsets increases, $\boldsymbol{\Sigma}$ becomes more diagonal, which results in higher GDOP and subsequently less MG, according to (24). In summary, by increasing the number of independent subsets, DG increases and MG decreases. Subsequently, by decreasing the number of independent subsets, DG decreases and MG increases. This means that there is an obvious tradeoff between DG and MG. Accordingly, the two following cases can be considered.

- First, assume that the links in each of the $\zeta$ subsets are correlated (maybe partially correlated) with each other and uncorrelated (maybe partially independent) from the ones in another subset. In this case, the optimal diversity order which is $\zeta$ is gained, when the links in each subset are completely independent of the ones in another subset resulting in $\zeta$ number of completely independent subsets. Any other case results in less diversity gain.

- Next, assume the same case, i.e. the links in each of the $\zeta$ subsets are correlated (maybe partially correlated) with each other and uncorrelated (maybe partially independent) from the ones in another subset. In this case, the system reaches the optimal multiplexing gain when the links in each subset are fully correlated. Any other case, in which the links in a subset are not fully correlated, results in less multiplexing gain.

In this way, the optimal tradeoff can be inferred: for each pair of transmit-receive links, the corresponding target's RCSs should be completely independent or fully correlated. In such optimal case, the diversity order equals the number of independent subsets, i.e., $\zeta$.

\section{Simulations}

In this part, we study the validity of the previous theoretical achievements through simulations.

Consider a MIMO radar system with three transmit antennas and three receive antennas that should be placed on the circumference of a circle with diameter of $10 \mathrm{~km}$. Therefore, nine $(3 \times 3=9)$ different RCS values (or equivalently nine different signal links from the transmitters to the receivers) are seen at the receivers. If the corresponding pairs of transmitreceive antennas of two paths are separated enough in space, the resulted RCS values will be uncorrelated, otherwise fully correlated. As mentioned before, our chosen model for becoming uncorrelated is based on the conditions mentioned in [2]. The target is posited at the center of the circle and the transmitted signals have equal powers. Next, we examine the spatial MIMO gains for different configurations of the aforementioned antennas:

1. All six antennas are placed at the same position on the circle's circumference resulting in fully correlated links.

2. All six antennas are placed separately enough, so that all resulted RCS values are completely independent. 
3. Two transmit antennas are placed at the same position and other antennas are placed separately enough, so that six subsets (with $\{2,2,2,1,1,1\}$ number of members in each one) can be constructed, that the links in each subset are fully correlated with each other, but completely independent of the links of other subsets.

4. The three transmit antennas are placed at the same position, and other antennas are placed separately enough, so that three subsets (with $\{3,3,3\}$ number of members in each one) can be constructed, that the links in each subset are fully correlated, but completely independent of others.

These configurations are shown in Fig. 1. Figure 2 depicts the resulting probability of missed detection for these four configurations as a function of SNR. The resolution capability of each configuration, expressed in terms of GDOP, versus SNR can be seen in Fig. 3.
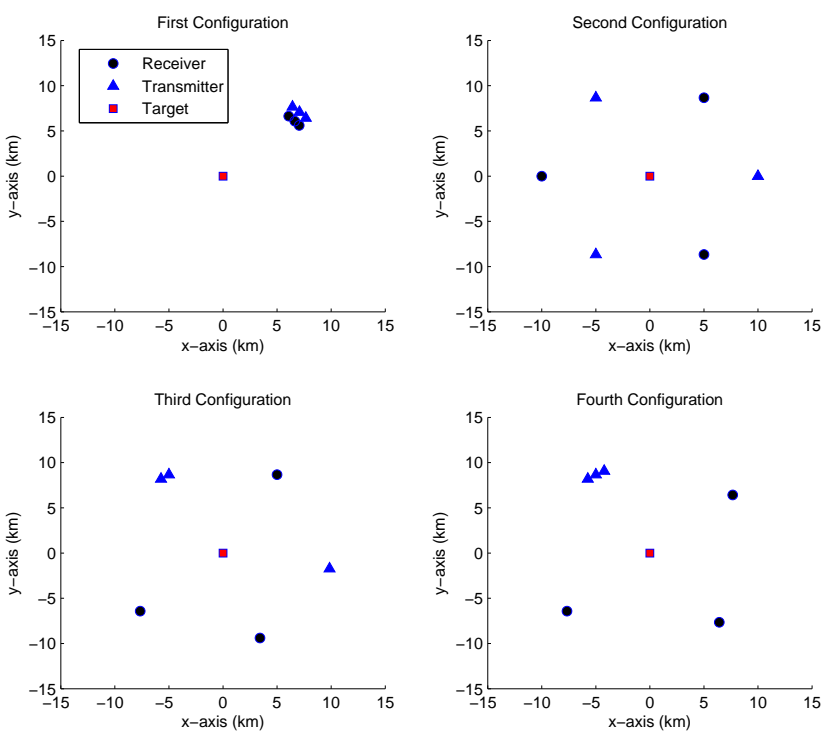

Fig. 1. Scenario configurations.

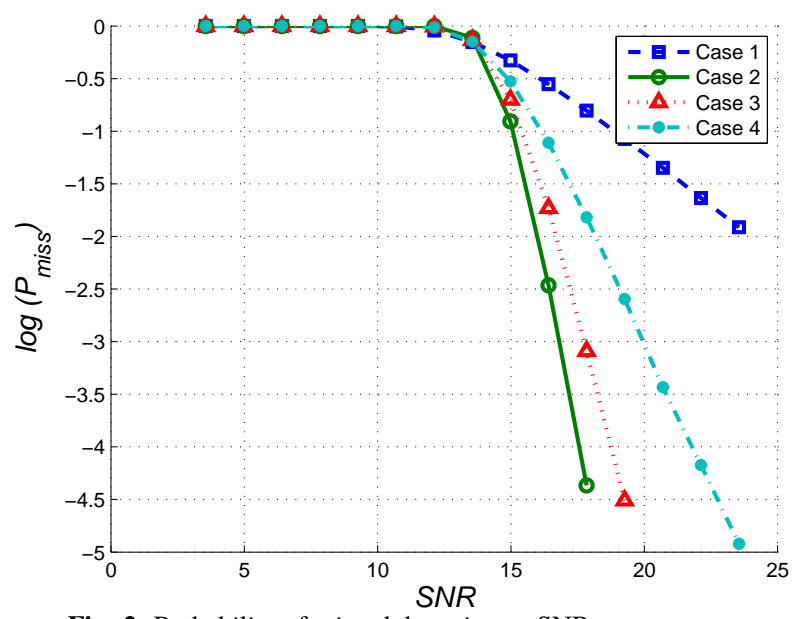

Fig. 2. Probability of missed detection vs SNR.

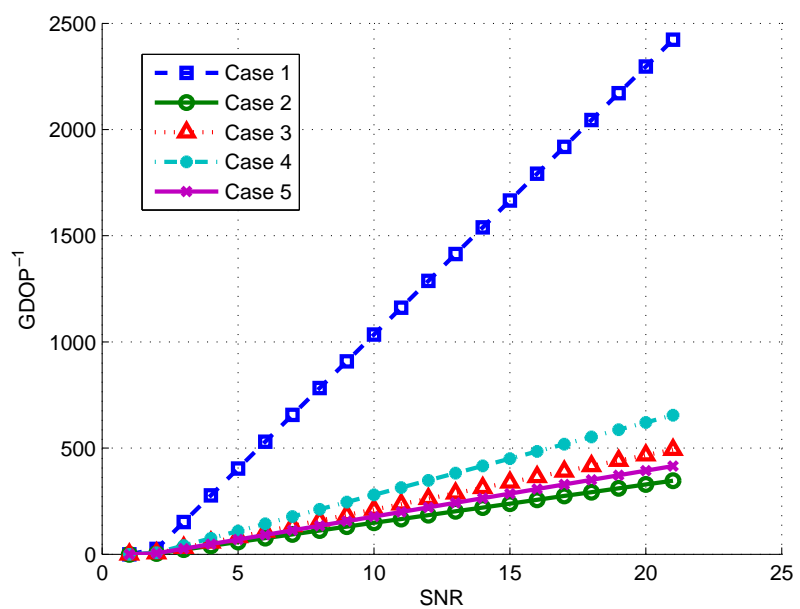

Fig. 3. Localization accuracy vs SNR.

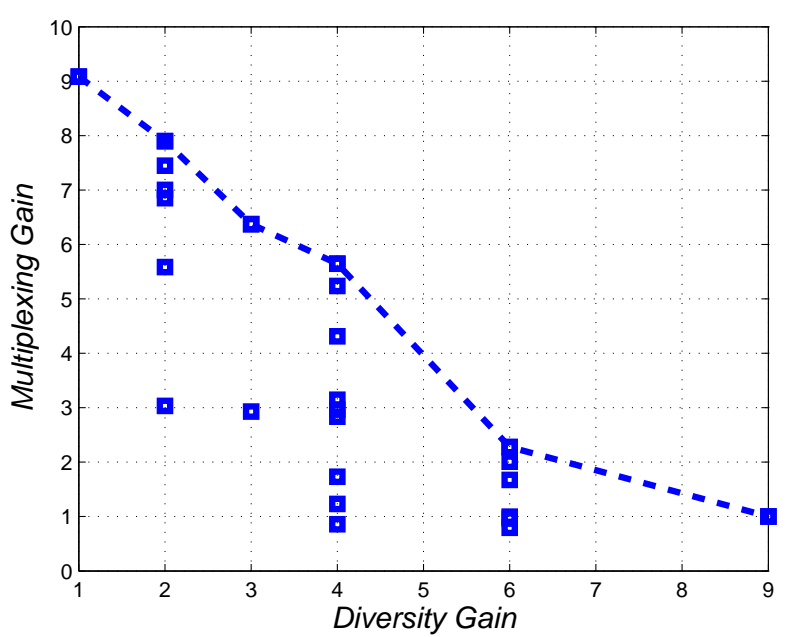

Fig. 4. Optimal diversity-multiplexing tradeoff in a $3 \times 3$ WSMIMO radar.

As expected, the second configuration results in maximum diversity gain. This fact can be seen in Fig. 2, as the slope of its diagram has the largest value. Besides, the first configuration results in minimum diversity gain, as expected too.

In addition, as shown in Fig. 3, the GDOP diagram's slope of the first configuration adopts the highest value and the slope of the second configuration adopts the lowest value, that verifies the previous theoretical results. In both figures of 2 and 3, the third and fourth configurations achieve diversity and multiplexing gains between the two previous extreme bounds.

Finally, consider a fifth scenario, in which two transmit antennas are placed near each other and other antennas are placed separately enough, such that the nine links can be subdivided into six subsets of $\{2,2,2,1,1,1\}$ members. The links in each subset are partially correlated with each other (not fully), but completely independent of others. In this case, the systems' multiplexing gain can be considered as the slope of the line corresponding to the 'case 5' in Fig. 3. As expected, the multiplexing gain attained in this case is less than the gain of cases 1,3 , and 4 . This simulation verifies the theoretical result previously obtained in Sec. 5. There, 
it was stated that in order to reach the optimal multiplexing gain, the links in a subset should be fully correlated.

In Fig. 4, the diversity gain and multiplexing gain of all possible cases of a $3 \times 3$ WS-MIMO radar are shown according to (20) and (24). As can be seen, the dashed line represents the optimal diversity-multiplexing gain tradeoff, and the points below such line show the gains corresponding to non-optimal cases.

\section{Conclusion}

In this paper, we investigated the tradeoff between two key gains of a MIMO radar with widely separated antennas: spatial diversity gain and spatial multiplexing gain. In particular, we demonstrated that there is interplay between the improvement that appears in the probability of missed detection due to the diversity of target's RCS and the resolution capability of a MIMO radar as a representative of its multiplexing gain value. In addition, we obtained the optimal diversity-multiplexing tradeoff, in which the MIMO radar system reaches its best performance from the perspective of exploiting the MIMO gains. We showed that in order to achieve such optimal case, each pair of transmit-receive links should be completely independent or fully correlated.

\section{References}

[1] LI, J., STOICA, P. MIMO radar with colocated antennas. IEEE Signal Processing Magazine, 2007, vol. 24, no. 5, p. 106-114. DOI: 10.1109/MSP.2007.904812

[2] FISHLER, E., HAIMOVICH, A., BLUM, R. S., et al. Spatial diversity in radars-models and detection performance. IEEE Transactions on Signal Processing, 2006, vol. 54, no. 3, p. 823-838. DOI: 10.1109/TSP.2005.862813

[3] LEHMANN, N., FISHLER, E., HAIMOVICH, A., et al. Evaluation of transmit diversity in MIMO-radar direction finding. IEEE Transactions on Signal Processing, 2007, vol. 55, no. 5, p. 2215-2225. DOI: 10.1109/TSP.2007.893220

[4] RADMARD, M., NAYEBI, M. M., KARBASI, S. M. Diversity-based geometry optimization in MIMO passive coherent location. Radioengineering, 2014, vol. 23 , no. 1, p. 41-49. ISSN: 1805-9600

[5] FISHLER, E., HAIMOVICH, A., BLUM, R. S., et al. MIMO radar: An idea whose time has come. In Proceedings of the IEEE Radar Conference, 2004, p. 71-78. DOI: 10.1109/NRC.2004.1316398

[6] RADMARD, M., NAYEBI, M. M. Target tracking and receiver placement in MIMO DVB-T based PCL. Iranian Journal of Science and Technology, Transactions of Electrical Engineering. [In press] Cited: 2016-09-06.

[7] HAIMOVICH, A., BLUM, R. S., CIMINI, L. J. MIMO radar with widely separated antennas. IEEE Signal Processing Magazine, 2008, vol. 25, no. 1, p. 116-129. DOI: 10.1109/MSP.2008.4408448

[8] RADMARD, M., KARBASI, S. M., NAYEBI, M. M. Data fusion in MIMO DVB-T -based passive coherent location. IEEE Transactions on Aerospace and Electronic Systems, 2013, vol. 49, no. 3, p. 1725-1737. DOI: 10.1109/TAES.2013.6558015
[9] ZHENG, L., TSE, D. Diversity and multiplexing: A fundamental tradeoff in multiple-antenna channels. IEEE Transactions on Information Theory, 2003, vol. 49, no. 5, p. 1073-1096. DOI: 10.1109/TIT.2003.810646

[10] KIM, T. T., SKOGLUND, M. Diversity-multiplexing tradeoff in MIMO channels with partial CSIT. IEEE Transactions on Information Theory, 2007, vol. 53, no. 8, p. 2743-2759. DOI: 10.1109/TIT.2007.901171

[11] JIANG, Y., VARANASI, M. K. Diversity-multiplexing tradeoff of MIMO systems with antenna selection. In Proceedings of the IEEE International Symposium on Information Theory (ISIT). 2007, p. 28362840. DOI: 10.1109/ISIT.2007.4557648

[12] GARCIA-ORDONEZ, L., PAGES-ZAMORA, A., FONOLLOSA, J. R. Diversity and multiplexing tradeoff of spatial multiplexing MIMO systems with CSI. IEEE Transactions on Information Theory, 2008, vol. 54, no. 7, p. 2959-2975. DOI: 10.1109/TIT.2008.924670

[13] KARMAKAR, S., VARANASI, M. K. The diversity-multiplexing tradeoff of the dynamic decode-and-forward protocol on a MIMO half-duplex relay channel. IEEE Transactions on Information Theory, 2011, vol.57, no. 10, p. 6569-6590. DOI: 10.1109/TIT.2011.2165828

[14] DAHER, R., ADVE, R. A notion of diversity order in distributed radar networks. IEEE Transactions on Aerospace and Electronic Systems, 2010, vol. 46, no. 2, p. 818-831. DOI: 10.1109/TAES.2010.5461659

[15] DE MAIO, A., LOPS, M., VENTURINO, L. Diversityintegration tradeoffs in MIMO detection. IEEE Transactions on Signal Processing, 2008, vol. 56, no. 10, p. 5051-5061. DOI: 10.1109/TSP.2008.928693

[16] HE, Q., BLUM, R. S. Diversity gain for MIMO Neyman-Pearson signal detection. IEEE Transactions on Signal Processing, 2011, vol. 59, no. 3, p. 869-881. DOI: 10.1109/TSP.2010.2094611

[17] VAN TREES, H. L. Detection, Estimation, and Modulation Theory, Part l: Detection, Estimation, and Linear Modulation Theory. John Wiley and Sons, 2001. ISBN: 978-0-471-46382-5

[18] MOSCHOPOUlOS, P. G., CANADA, W. B. The distribution function of a linear combination of chi-squares. Computers and mathematics with applications, 1984, vol. 10, no. 4, p. 383-386. DOI: 10.1016/0898-1221(84)90066-X

[19] SKOLNIK, M. I. Introduction to Radar. 3rd ed., McGraw-Hill, 2001. ISBN: 0-07-044533-8

[20] GODRICH, H., HAIMOVICH, A., BLUM, R. Target localisation techniques and tools for multiple-input multiple-output radar. IET Radar, Sonar and Navigation, 2009, vol. 3, no. 4, p. 314-327. DOI: 10.1049 /ietrsn.2008.0141

\section{About the Authors ...}

Mohammad NAZARI MAJD received B.Sc.Eng and M.Sc.Eng degrees in the communications field from Sharif University Technology, Tehran, Iran. He was approved as top talent in both B.Sc. and M.Sc. from the university. He is currently working toward his Ph.D. degree at the same university. His research interests include MIMO radars, noise radars, detection theory, and radar signal processing. He was a member of the Iranian Physics Olympiad team in 2008.

Mojtaba RADMARD received the B.S., M.S. and PhD degrees in electrical engineering, all from Sharif University of Technology, Tehran, Iran. He is now working as a postdoc researcher at the same university. He got the "Shahid Chamran" prize for postdoctoral researchers from the Iran's 
National Elites Foundation in 2014. His research interests include MIMO systems, tracking, passive coherent location, radar signal processing and speech processing.

Mohammad Mahdi CHITGARHA received his B.Sc and M.Sc. degrees in the communications field from Sharif University of Technology, Tehran, Iran. He was ranked first in both M.Sc. and Ph.D. national university entrance exams. He is now a Ph.D. candidate at the same university. His research interests include MIMO radars, radar signal processing, detection theory, and speech signal processing.

Mohammad Hasan BASTANI received his B.Sc. degree in electrical engineering in 1979 from Sharif University of Technology, Tehran, Iran. He received his diplome dingenieur and doctoral degrees from ENST (Ecole National
Superieur de Telecommunications), Paris, France, in electrical engineering, in 1981 and 1984, respectively. He joint the faculty of department of electrical engineering of Sharif University in 1984. His research interests are in stochastic signal processing, data fusion, and radar design.

Mohammad Mahdi NAYEBI received the B.S. and M.S. degrees in 1988 and 1990, respectively, both from Sharif University of Technology, Tehran, Iran, with the honor of first rank. He received the Ph.D. degree in electrical engineering in 1994 from Tarbiat Modarres University, Tehran, Iran, with the same honor. He joined the Sharif University of Technology faculty in 1994, and became an Associate Professor in 1998, and Professor in 2003. His main research interests are radar signal processing and detection theory. 